\title{
Generations of Correlation Averages
}

\author{
Giovanni Coppola and Maurizio Laporta \\ Dipartimento di Matematica e Applicazioni, Università degli Studi di Napoli, Via Cinthia, 80126 Napoli, Italy
}

Correspondence should be addressed to Maurizio Laporta; mlaporta@unina.it

Received 5 March 2014; Accepted 28 April 2014; Published 18 June 2014

Academic Editor: Aloys Krieg

Copyright (C) 2014 G. Coppola and M. Laporta. This is an open access article distributed under the Creative Commons Attribution License, which permits unrestricted use, distribution, and reproduction in any medium, provided the original work is properly cited.

We give a general link between weighted Selberg integrals of any arithmetic function $f$ and averages of $f$ correlations in short intervals, proved by the elementary dispersion method (our version of Linnik's method). We formulate conjectural bounds for the so-called modified Selberg integral of the divisor functions $d_{k}(n)$, gauged by the Cesaro weight in the short interval $n \in[x-H, x+H]$ and improved by these some recent results by Ivić. The same link provides, also, an unconditional improvement. Then, some remarkable conditional implications on the $2 k$ th moments of Riemann zeta function on the critical line are derived. We also give general requirements on $f$ that allow our treatment for $f$ weighted Selberg integrals.

\section{Introduction and Statement of the Results}

In the milestone paper [1], Selberg introduced an important tool in the study of the distribution of prime numbers in short intervals $[x, x+H]$ with $H=o(x)$ as $x \rightarrow \infty$, that is, the integral

$$
\int_{N}^{2 N}\left|\sum_{x<n \leq x+H} \Lambda(n)-H\right|^{2} \mathrm{~d} x
$$

where $\Lambda$ is the von Mangoldt function: $\Lambda(n) \stackrel{\text { def }}{=} \log p$ if $n=p^{r}$ for some prime $p$ and $r \in \mathbb{N}$; otherwise, $\Lambda(n) \stackrel{\text { def }}{=}$ 0 . Thus, $\Lambda(n)$ is a weighted characteristic function of prime numbers generated by $-\zeta^{\prime}(s) / \zeta(s)$ (hereafter, $\zeta$ is the Riemann zeta function). The Selberg integral, being a quadratic mean, pertains precisely to the study of the distribution of primes in almost all short intervals $[x, x+H]$, that is, with at most $o(N)$ exceptional integers $x \in[N, 2 N]$ as $N \rightarrow \infty$. Here, we define the Selberg integral of any $f: \mathbb{N} \rightarrow \mathbb{C}$ as

$$
J_{f}(N, H) \stackrel{\text { def }}{=} \sum_{x \sim N}\left|\sum_{x<n \leq x+H} f(n)-M_{f}(x, H)\right|^{2},
$$

where $x \sim N$ means $N<x \leq 2 N$ and $M_{f}(x, H)$ is the expected mean value of $f$ in short intervals (abbreviated as s.i. mean value). In order to avoid trivialities, we assume that $H$ goes to infinity with $N$. In view of nontrivial bounds, the discrete version $J_{\Lambda}(N, H)$ may be considered close enough to the original continuous integral; so, we are legitimate to use the same symbol for both. Similar considerations hold for the $f$ we work with, mostly the $k$-divisor function $d_{k}$ for $k \geq 3$, where $d_{k}(n)$ is the number of ways to write $n$ as a product of $k$ positive integers (see [2] and compare Section 4). Let us denote the Selberg integral of $d_{k}$ as

$$
J_{k}(N, H) \stackrel{\text { def }}{=} \sum_{x \sim N}\left|\sum_{x<n \leq x+H} d_{k}(n)-M_{k}(x, H)\right|^{2}
$$

with the s.i. mean value of $d_{k}$ given by

$$
M_{k}(x, H) \stackrel{\text { def }}{=} H\left(P_{k-1}(\log x)+P_{k-1}^{\prime}(\log x)\right),
$$

where $P_{k-1}$ is the residual polynomial of degree $k-1$ such that $P_{k-1}(\log x) \stackrel{\text { def }}{=} \operatorname{Res}_{s=1} \zeta^{k}(s) x^{s-1} / s$.

The first author [2] has proved the lower bound $N H L^{k+1} \ll_{k} J_{k}(N, H)$ for $N^{\varepsilon} \ll H \ll N^{1 / k-\varepsilon}$, where 
$L \stackrel{\text { def }}{=} \log N$ hereafter. We formulate, for the so-called modified Selberg integral of $d_{3}$,

$$
\begin{aligned}
& \widetilde{J}_{3}(N, H) \\
& \stackrel{\text { def }}{=} \sum_{x \sim N}\left|\sum_{0 \leq|n-x| \leq H}\left(1-\frac{|n-x|}{H}\right) d_{3}(n)-M_{3}(x, H)\right|^{2},
\end{aligned}
$$

where $M_{3}(x, H)$ is the same s.i. mean value as in $J_{3}(N, H)$, the following conjecture. In Section 7, Propositions 16 and 18 justify the admissibility of such a choice for the s.i. mean value in modified Selberg integrals, even in the generalization for any divisor function $d_{k}$ (everything comes from Proposition 14; see Section 7).

Hereafter, we abbreviate $A(N, H) \lll B(N, H)$ whenever $A(N, H) \ll_{\varepsilon} N^{\varepsilon} B(N, H)$, for all $\varepsilon>0$.

Conjecture CL. If $H \ll N^{1 / 3}$, then $\widetilde{J}_{3}(N, H) \lll N H$. result.

A first consequence of our conjecture is the following

Theorem 1. If Conjecture CL holds, then $J_{3}(N, H) \lll N H^{3 / 2}$.

In Section 5, we deduce this from a general link (Lemma 10), between the Selberg integral $J_{f}(N, H)$ and the corresponding modified Selberg integral,

$$
\begin{aligned}
& \widetilde{J}_{f}(N, H) \\
& \stackrel{\text { def }}{=} \sum_{x \sim N}\left|\sum_{0 \leq|n-x| \leq H}\left(1-\frac{|n-x|}{H}\right) f(n)-M_{f}(x, H)\right|^{2},
\end{aligned}
$$

in the special case of an essentially bounded (see Section 4) real function $f$ such that $M_{f}(x, H)$ vanishes identically.

Moreover, for the width of $H$, that is, $\theta \stackrel{\text { def }}{=} \log H / \log N$ (more generally, $\theta$ is defined by $x^{\theta} \ll H \ll x^{\theta}$ for $x \sim N$ ), we will implicitly assume that any inequality $\theta>\theta_{0}$ (resp., $\left.\theta<\theta_{0}\right)$ means that $\exists \delta>0$ fixed and absolute constant such that $\theta \geq \theta_{0}+\delta$ (resp., $\theta \leq \theta_{0}-\delta$ ). In particular, $0<\theta<1$ works as $\delta \leq \theta \leq 1-\delta$.

Noteworthy, Theorem 1 improves Ivić's results [3] for $d_{3}$, both in the bound and in the low range, while Ivić's bound is nontrivial for $N^{1 / 6+\delta} \ll H \ll N^{1-\delta}$, that is, for width $1 / 6<\theta<1$; ours is nontrivial for $0<\theta \leq 1 / 3$, that is, for $N^{\delta} \ll H \ll N^{1 / 3}$ (we think to be able to get a wider range). Remarkably, under Conjecture CL, the first author has recently derived the even better $J_{3}(N, H) \lll N H^{6 / 5}$ (see http://arxiv.org/).

As one may expect, our study applies to every divisor function $d_{k}$, though the conjectured estimate for

$$
\begin{aligned}
& \widetilde{J}_{k}(N, H) \\
& \stackrel{\text { def }}{=} \sum_{x \sim N}\left|\sum_{0 \leq|n-x| \leq H}\left(1-\frac{|n-x|}{H}\right) d_{k}(n)-M_{k}(x, H)\right|^{2}
\end{aligned}
$$

becomes less and less meaningful as $k$ grows: this is due to known bounds for $\alpha_{k}$ in (compare Section 4)

$$
\sum_{n \leq x} d_{k}(n)-x P_{k-1}(\log x) \lll x^{\alpha_{k}} .
$$

We generalize Conjecture CL for the modified Selberg integral of $d_{k}$ with $k \in\{4,5,6,7\}$ as follows.

General Conjecture CL. Fix $k \in\{4,5,6,7\}$ and assume that $\alpha_{k-1} \in[0,1)$ in $(*)$. If $H \ll N^{1 / 2}$, then

$$
\widetilde{J}_{k}(N, H) \lll N^{1-1 / k} H^{2}+N H\left(N^{1-4 / k}+N^{(1-1 / k) \alpha_{k-1}-1 / k}\right) .
$$

Consequently, setting $\widetilde{\theta}_{k} \stackrel{\text { def }}{=} \max \left(1-4 / k,(1-1 / k) \alpha_{k-1}-1 / k\right)$, for every width $\theta \in\left(\widetilde{\theta}_{k}, 1 / 2\right]$, there exists an $\varepsilon_{1}=\varepsilon_{1}(\theta, k)>0$ such that

$$
\widetilde{J}_{k}(N, H) \ll_{k} N^{1-4 \varepsilon_{1}} H^{2} .
$$

From the last inequality, our second main result follows, proved together with Theorem 1 in Section 5.

Theorem 2. If General Conjecture CL holds, then $J_{k}(N, H) \ll_{k} N^{1-2 \varepsilon_{1}} H^{2}$ for $k \in\{4,5,6,7\}$.

As a consequence, one would get an improvement in the low range of $H$ with respect to the results of Ivić for the meansquare of $d_{k}$ in short intervals [3]. In fact, Theorem 1 in [3] holds for $\theta \in\left(\theta_{k}, 1\right)$ with $\theta_{k}=\theta(k)$ defined in terms of Carlson's abscissa $\sigma_{k}$ (see Section 6). In particular, it holds for $\theta_{4}=1 / 4, \theta_{5}=11 / 30$, and $\theta_{6}=3 / 7$, whereas from $\alpha_{k}$ bounds [4], we get instead $\widetilde{\theta}_{k}<\theta \leq 1 / 2$ with $\widetilde{\theta}_{4}=11 / 128, \widetilde{\theta}_{5}=1 / 5$, $\widetilde{\theta}_{6}=1 / 3$.

More generally, $\widetilde{J}_{f}(N, H)$ is a kind of smoothing (see Section 2) for the Selberg integral $J_{f}(N, H)$, both in the arithmetic and in the harmonic analysis aspects. The arithmetic one goes back to Ernesto Cesaro:

$$
\sum_{0 \leq|n-x| \leq H}\left(1-\frac{|n-x|}{H}\right) f(n)=\frac{1}{H} \sum_{h \leq H} \sum_{|n-x|<h} f(n) .
$$

This arithmetic mean of the inner sum in $J_{f}(N, H)$ justifies the choice of the same $M_{f}(x, H)$ in $\widetilde{J}_{f}(N, H)$. The analytic aspects of such a smoothing process will be clear after the introduction of the correlation $\mathscr{C}_{f}(h)$ in Section 3 , where it is shown that Selberg integrals $J_{w, f}(N, H)$ are averages of $\mathscr{C}_{f}(h)$ in short intervals $|h| \ll H$. In fact (see Section 3 title), a kind of elementary dispersion method proves this link.

The next corollaries, applying such an intimate link, improve recent results $[5,6]$ on an additive divisor problem for $d_{k}$ (first two conditionally, third unconditionally). They concern the deviation of $d_{k}$; that is,

$$
\mathbb{D}_{k}(N, H) \stackrel{\text { def }}{=} \sum_{h \leq H} \mathscr{C}_{k}(h)-H \sum_{n \sim N} p_{k-1}(\log n)^{2},
$$

where $\mathscr{C}_{k}(h) \stackrel{\text { def }}{=} \sum_{n \sim N} d_{k}(n) d_{k}(n-h)$ is the correlation (see Section 3) of $d_{k}$ and $p_{k-1}(\log n) \stackrel{\text { def }}{=} M_{k}(n, H) / H$ is 
the so-called logarithmic polynomial of $d_{k}(n)$. The following consequence of Theorem 1 is proved in Section 6 .

Corollary 3. Let $N, H \in \mathbb{N}$ be such that $N^{\theta} \ll H \ll N^{\theta}$ for a fixed $\theta \in(0,1 / 3]$. If Conjecture $C L$ is true, then

$$
\mathbb{D}_{3}(N, H) \lll N H^{3 / 4}+N^{\alpha_{3}} H+H^{2} .
$$

In a completely analogous way, from Theorem 2 , we deduce the following result on $\mathbb{D}_{k}$ for $k \in\{4,5,6,7\}$.

Corollary 4. Under the same hypotheses of Theorem 2, one has, in the same ranges and for the same $\varepsilon_{1}$,

$$
\mathbb{D}_{k}(N, H) \lll N^{1-\varepsilon_{1}} H .
$$

The aforementioned link to $[5,6]$ results relies on the identity (see Section 4)

$$
p_{k-1}(\log x)=\operatorname{Res}_{s=1} \zeta^{k}(s) x^{s-1}
$$

where one has to be acquainted that one's notations $P_{k-1}$ and $p_{k-1}$ are not consistent with those in $[5,6]$. In particular, from (3.8) in [6], the main term in the $[5,6]$ formulas for sums of $d_{k}$ correlations is

$$
H \int_{N}^{2 N} p_{k-1}(\log x)^{2} \mathrm{~d} x+O_{\varepsilon}\left(N^{1+\varepsilon}\right) .
$$

Since it is easily seen that, for every $H \ll N$, one has

$$
H \sum_{n \sim N} p_{k-1}(\log n)^{2}-H \int_{N}^{2 N} p_{k-1}(\log x)^{2} \mathrm{~d} x \lll N,
$$

then our $\mathbb{D}_{k}(N, H)$ is comparable with the $\Delta_{k}(N ; h)$ average for $d_{k}$ correlations, estimated in [5] when $k=3$ and in [6] for every $k \geq 3$. Thus, Corollary 3 and the bound $\alpha_{3} \leq 43 / 96$ (see [4]) imply $\mathbb{D}_{3}(N, H) \lll N H^{3 / 4}$ for $\theta \leq 1 / 3$, improving [5] in the low range of short intervals (while [6] bounds are better for $k=3$ if $\theta>1 / 2$ ); in fact, their remainders $\lll N^{13 / 12} H^{1 / 2}$ (worse than ours when $\theta<1 / 3$ ) are nontrivial only for $\theta>$ $1 / 6$. Similarly, since $\alpha_{k}<1$, Corollary 4 improves [6] bounds for the low range (but not for $1 / 2<\theta<1$ ).

Our Lemma 12 (see Remark 13, Section 5) and Ivić's Theorem 1 [3] prove the following unconditional result.

Corollary 5. For a fixed integer $k \geq 3$, let $\Delta_{k}(N ; h)$ be defined as in [6] and for a fixed $\theta>2 \sigma_{k}-1$, where $\sigma_{k}$ is Carlson's abscissa, let $N^{\theta} \ll H \ll N^{\theta}$ as $N \rightarrow \infty$. Then

$$
\sum_{h \leq H} \Delta_{k}(N ; h) \ll_{\varepsilon} N^{1-\varepsilon} H,
$$

where $\varepsilon=\varepsilon(k, \theta)$ might depend also on $\varepsilon_{1}(k)>0$ given in [3].

The novelty of our approach is that, though conditionally, it improves the analogous achievements obtained via the classical moments of the Riemann zeta function on the critical line (a major example being the known upper bounds for Carlson's abscissa in Ivić [3] results). We think that our conjectures might be approachable by elementary arguments, starting with the so-called $k$-folding method (Section 7 , Proposition 14).

On the other hand, estimates for $J_{k}(N, H)$ have nontrivial consequences on the $2 k$ th moments of the Riemann $\zeta$ function on the critical line (see [7]):

$$
I_{k}(T) \stackrel{\text { def }}{=} \int_{T}^{2 T}\left|\zeta\left(\frac{1}{2}+i t\right)\right|^{2 k} \mathrm{~d} t .
$$

At present, we content ourselves with having found an alternative way to pursue possible improvements on the $2 k$ th moments of $\zeta$, for at least relatively low values of $k$. Indeed, in Section 8, we take a glance at the effect of hypothetical estimates for Selberg integrals on the $2 k$ th moments, through Theorem 1.1 of [7], whereas the first author (see http://arxiv.org/) provides the best known estimate for the 6th moment (under CL).

In Section 8, we prove next link, between $I_{k}(T)$ bounds and $J_{k}(N, H)$ bounds (compare [8] conditional bounds).

Theorem 6. Let $k \geq 3$ be fixed. If $J_{k}(N, H) \lll N^{1+A} H^{1+B}$ holds for $H \ll N^{1-2 / k}$ and for some constants $A, B \geq 0$, then $I_{k}(T) \lll T^{1+k(A+B) / 2-B}$ (beside the $\varepsilon$ dependence, implicit constant may depend on $k$ ).

This result encourages us to seek nontrivial bounds for the $d_{k}$ Selberg integrals, in the future.

As already said, we will give some Propositions, besides above results; we will not prove them here. We state Proposition 9 (about proximity of two different forms of $d_{3}$ expected value in short intervals) in Section 4, while Propositions 14,16 , and 18 (about, resp., the $k$-folding, the nontrivial arithmetic bound of $d_{k}$ weighted Selberg integrals, and the mean-square proximity of $d_{k}$ expected value in s.i., for all $k \geq 3$ ) are stated in Section 7.

We will provide the necessary Lemmas for the proof of our results in due course, in next sections. In particular, we prove Lemma 7 (our elementary version of Linnik's method) in Section 3 and Lemma 10 (linking 2nd and 3rd generation) and Lemma 12 (linking 1st and 2nd generation) in Section 5.

1.1. Some Notation and Conventions. If the implicit $O$ and $\ll$ constants depend on parameters like $\varepsilon>0$, mostly, we write $\mathrm{O}_{\varepsilon}$ and $\ll_{\varepsilon}$, but we omit subscripts for the « symbol. As usual, $\varepsilon>0$ is arbitrarily small, changing from statement to statement. The relation $f \sim g$ means that $f=g+o(g)$ as the main variable tends to infinity typically. There is no confusion with the dyadic notation, $x \sim N$, that means $x \in(N, 2 N] \cap \mathbb{Z}$. The Möbius function is $\mu(1) \stackrel{\text { def }}{=} 1, \mu(n) \stackrel{\text { def }}{=}(-1)^{r}$ if $n$ is the product of $r$ distinct primes, and $\mu(n) \stackrel{\text { def }}{=} 0$ otherwise. Symbol $\mathbf{1}$ denotes constant 1 function and $\mathbf{1}_{U}$ is the $U$ characteristic function. The Dirichlet convolution product of the arithmetic functions $f_{1}$ and $f_{2}$ is $\left(f_{1} * f_{2}\right)(n) \stackrel{\text { def }}{=} \sum_{d \mid n} f_{1}(d) f_{2}(n / d)$. The $k$-fold Dirichlet product of $f$ is $\underbrace{f * \cdots * f}_{k \text { times }}$, so $d_{k} \stackrel{\text { def }}{=}$ $\underline{1 * \cdots * 1}$, for all $k \geq 2$. For any $f, g: \mathbb{N} \rightarrow \mathbb{C}$, we call $g$ $k$ times 
the Eratosthenes transform of $f$ if $g=f * \mu$ or equivalently $f=g * 1$ (Möbius' inversion formula): $\mathbf{1}$ is the Eratosthenes transform of $\mathbf{d} \stackrel{\text { def }}{=} \mathbf{1} * \mathbf{1}$, the divisor function. We omit $a \geq 1$ in sums like $\sum_{a \leq X}$. The distance of $\alpha \in \mathbb{R}$ from $\mathbb{Z}$ is $\|\alpha\| \stackrel{\text { def }}{=}$ $\min (\{\alpha\}, 1-\{\alpha\})$ and $\{\alpha\}$ is the fractional part of $\alpha$. As usual, $e(\alpha) \stackrel{\text { def }}{=} e^{2 \pi i \alpha}$, for all $\alpha \in \mathbb{R}$, and $e_{q}(a) \stackrel{\text { def }}{=} e(a / q)$, for all $q \in \mathbb{N}$, for all $a \in \mathbb{Z}$.

\section{Introducing Weighted Selberg Integrals}

Given positive integers $N$ and $H=o(N)$, the $w$-Selberg integral of an arithmetic function $f: \mathbb{N} \rightarrow \mathbb{C}$ is the weighted quadratic mean

$$
J_{w, f}(N, H) \stackrel{\text { def }}{=} \sum_{x \sim N}\left|\sum_{n} w(n-x) f(n)-M_{f}(x, w)\right|^{2},
$$

where the complex valued weight $w$ has support in $[-c H, c H]$ for some fixed real number $c>0$, so that the inner sum is genuinely finite. The term $M_{f}(x, w)$ is the expected mean value of $f$ weighted with $w$ in the short interval of length $\ll H$ and depends on $w$, when $f$ has logarithmic polynomial $p_{f}(\log n)$; see Section 4; set

$$
\begin{aligned}
M_{f}(x, w) & \stackrel{\text { def }}{=} \sum_{n} w(n-x) p_{f}(\log n) \\
& =p_{f}(\log x) \sum_{h} w(h)+O\left(\frac{H^{2} L^{c-1}}{N}\right) .
\end{aligned}
$$

Here, $c=\operatorname{deg}\left(p_{f}\right)$, with the convention that constant $p_{f} \neq 0$ has degree $c=0$, while $p_{f}=0$ has $c=-1$.

Clearly, these $J_{w, f}$ include the most celebrated Selberg integral $J_{\Lambda}(N, H)=J_{u, \Lambda}(N, H)$, with $u \stackrel{\text { def }}{=} \mathbf{1}_{[1, H]}$ being the characteristic function of $[1, H]$; more generally, $J_{f}(N, H)$ is the $u$-Selberg integral of $f$, while the modified Selberg integral $\widetilde{J}_{f}(N, H)$ (introduced in [9]) is recognizable by taking the Cesaro weight; say

$$
C_{H}(t) \stackrel{\text { def }}{=} \max \left(1-\frac{|t|}{H}, 0\right) .
$$

Since

$$
C_{H}(t)=\frac{1}{H} \sum_{a \leq H-|t|} 1=\frac{1}{H} \sum_{\substack{a, b \leq H \\ b-a=t}} \sum_{1}=\frac{\mathscr{C}_{u}(t)}{H},
$$

where $\mathscr{C}_{u}$ is the correlation of the weight $u$ (see next Section 3), it follows that the Cesaro weight is the normalized correlation of $u$. We say that we smooth $J_{w, f}(N, H)$ when we get the modified $w$-Selberg integral of $f$ :

$$
\widetilde{J}_{w, f}(N, H) \stackrel{\text { def }}{=} J_{\widetilde{w}, f}(N, H),
$$

where the new weight $\widetilde{w}$ is the normalized correlation of $w$; that is,

$$
\widetilde{w}(h) \stackrel{\text { def }}{=} \frac{1}{H} \sum_{\substack{n \\ n-m=h}} \sum_{\substack{m \\ \text { na }}} w(n) \overline{w(m)}
$$

As a general strategy, from a nonpositive definite weight $w$ (with support of length $\ll H$ ), we smooth it; the new weight $\widetilde{w}=\mathscr{C}_{w} / H$ has a nonnegative exponential sum $\widehat{\mathscr{C}}_{w}(\beta) / H=$ $|\widehat{w}(\beta)|^{2} / H$ (see next Section 3).

Another important instance, studied by the first author (see [10-13]), is the symmetry integral

$$
J_{\text {sgn }, f}(N, H) \stackrel{\text { def }}{=} \sum_{x \sim N}\left|\sum_{0 \leq|n-x| \leq H} \operatorname{sgn}(n-x) f(n)\right|^{2},
$$

where $\operatorname{sgn}(0) \stackrel{\text { def }}{=} 0, \operatorname{sgn}(t) \stackrel{\text { def }}{=}|t| / t$ for $t \neq 0$, and $M_{f}(x, \operatorname{sgn})$ vanishes identically for every $f$. Such a study has been motivated by the link found by Kaczorowski and Perelli [14], between the classical Selberg integral and the symmetry properties of the prime numbers.

We will exploit the links between the Selberg integral $J_{f}(N, H)$, the symmetry integral $J_{\text {sgn, } f}(N, H)$, and the modified Selberg integral $\widetilde{J}_{f}(N, H)$ (also, within other weighted integrals) in the future (see http://arxiv.org/).

Finally, the considerations in Section 4 suggest that a satisfactory general theory on the weighted Selberg integrals may be built within the Selberg Class (see [15] and http://arxiv.org/abs/1205.1706 in which v3 refers to the arxiv paper with same title of present paper).

\section{Weighted Selberg Integrals Are Correlation Averages}

The correlation of an arithmetic function $f: \mathbb{N} \rightarrow \mathbb{C}$ is a shifted convolution sum of the form

$$
\mathscr{C}_{f}(h) \stackrel{\text { def }}{=} \sum_{n \sim N} f(n) \overline{f(n-h)}
$$

where $h \in[-N, N] \cap \mathbb{Z}$ is the shift. Observe that one may restrict $f$ to $[1,3 N] \cap \mathbb{N}$. Further, a correlation of shift $h$ is strictly related to a weighted count of $n, m \in(N, 2 N] \cap \mathbb{N}$ such that $n-m=h$; namely,

$$
\mathscr{C}_{f}(h)=\sum_{\substack{n \sim N \\ n-m=h}} \sum_{\substack{m \sim N\\}} f(n) \overline{f(m)}+O\left(|h| \cdot \max _{\ell \sim N}|f(\ell)|^{2}\right) .
$$

We use the last formula to define the correlation of a weight $w$ by neglecting the $\mathrm{O}$-term conveniently in the present context; namely,

$$
\mathscr{C}_{w}(h) \stackrel{\text { def }}{=} \sum_{\substack{a \\ b-a=h}} \sum_{\substack{b \\ a=}} w(b) \overline{w(a)} .
$$


The reason of such a different definition will be clarified after the next lemma, where we prove a strict connection between correlations and weighted Selberg integrals by applying an elementary dispersion method.

Lemma 7. Let $N, H$ be positive integers such that $H \rightarrow \infty$ and $H=o(N)$ as $N \rightarrow \infty$. For every uniformly bounded weight $w$ with support in $[-c H, c H]$ and every arithmetic function $f$, one has

$$
\begin{aligned}
J_{w, f}(N, H)= & \sum_{0 \leq|h| \leq 2 c H} \mathscr{C}_{w}(h) \mathscr{C}_{f}(h) \\
& -2 \Re\left(\sum_{n} f(n) \sum_{x \sim N} w(n-x) \overline{M_{f}(x, w)}\right) \\
& +\sum_{x \sim N}\left|M_{f}(x, w)\right|^{2}+O\left(H^{3}\|f\|_{\infty}^{2}\right),
\end{aligned}
$$

where $\|f\|_{\infty} \stackrel{\text { def }}{=} \max _{N-c H<n \leq 2 N+c H}|f(n)|$.

Proof. It is readily seen that, after expanding the square and exchanging sums, it suffices to show, say,

$$
\begin{aligned}
\Sigma & \stackrel{\text { def }}{=} \sum_{n} f(n) \sum_{m} \overline{f(m)} \sum_{x \sim N} w(n-x) \overline{w(m-x)} \\
& =\sum_{h} \mathscr{C}_{w}(h) \mathscr{C}_{f}(h)+O\left(H^{3}\|f\|_{\infty}^{2}\right) .
\end{aligned}
$$

Since we may clearly assume that $a \stackrel{\text { def }}{=} m-x$ and $b \stackrel{\text { def }}{=} n-x \in$ $[-c H, c H]$, we write

$$
\Sigma=\sum_{|h| \in[0,2 c H]} \sum_{\substack{n \\ n-m=h}} \sum_{m}(n) \overline{f(m)} \sum_{\substack{a, b \in[-c H, c H] \\ b-a=h \\ n-b=m-a \in(N, 2 N]}} w(b) \overline{w(a)} .
$$

By noting that the condition $n-b=m-a \in(N, 2 N]$ is implied by $n, m \in(N+c H, 2 N-c H]$, one has

$$
\begin{aligned}
\Sigma= & \sum_{|h| \in[0,2 c H]} \sum_{\substack{n, m \in(N+c H, 2 N-c H] \\
n-m=h}} f(n) \overline{f(m)} \\
& \times \sum_{\substack{a, b \in[-c H, c H] \\
b-a=h}} w(b) \overline{w(a)}
\end{aligned}
$$

$$
\begin{aligned}
& +O\left(H\|f\|_{\infty}^{2}\right. \\
& \times \sum_{|h| \in[0,2 c H]}\left(\sum_{n} \sum_{\substack{m \in(N-c H, N+c H] \cup(2 N-c H, 2 N+c H] \\
n-m=h}} 1\right. \\
& \left.\left.+\sum_{m} \sum_{n \in(N-c H, N+c H] \cup(2 N-c H, 2 N+c H]}^{n-m=h} 1\right)\right) \\
& =\sum_{|h| \in[0,2 c H]}\left(\sum_{\substack{n \sim N, m \in(N+c H, 2 N-c H] \\
n-m=h}} f(n) \overline{f(m)}\right. \\
& \left.+\sum_{\substack{n \in(N+c H, 2 N-c H], m \sim N \\
n-m=h}} f(n) \overline{f(m)}\right) \\
& \times \sum_{\substack{a, b \in[-c H, c H] \\
b-a=h}} w(b) \overline{w(a)} \\
& +O\left(H\|f\|_{\infty}^{2} \sum_{h \ll H} \sum_{m \in(N, N+c H] \cup(2 N-c H, 2 N]} \sum_{\substack{n-m= \pm h \\
1}} 1\right) \\
& +O\left(H\|f\|_{\infty}^{2} \sum_{h \ll H} \sum_{m \in(N-c H, N+c H] \cup(2 N-c H, 2 N+c H]} 1\right) \\
& =\sum_{0 \leq|h| \leq 2 c H} \sum_{\substack{n \sim N \\
n-m=h}} \sum_{m \sim N} f(n) \overline{f(m)} \sum_{\substack{-c H \leq a, b \leq c H \\
b-a=h}} w(b) \overline{w(a)} \\
& +O\left(H\|f\|_{\infty}^{2} \sum_{h \ll H} \sum_{m \in(N-c H, N+c H] \cup(2 N-c H, 2 N+c H]}^{1}\right) \\
& =\sum_{0 \leq|h| \leq 2 c H}\left(\mathscr{C}_{f}(h)+O\left(\|f\|_{\infty}^{2}|h|\right)\right) \mathscr{C}_{w}(h) \\
& +O\left(H^{2}\|f\|_{\infty}^{2}(2 c H+1)\right) \\
& =\sum_{h} \mathscr{C}_{w}(h) \mathscr{C}_{f}(h)+O\left(H^{3}\|f\|_{\infty}^{2}\right) .
\end{aligned}
$$

Remark 8. Essentially the remainder term $O\left(H^{3}\|f\|_{\infty}^{2}\right)$ comes from the estimate for short segments of length $\ll H$ within long sums of length $\gg N$. We refer to these short segments as the tails in the summations. To simplify our exposition, the symbol $(T)$ within some of the next formulas 
will warn the reader of some tails discarded to abbreviate the formulas themselves.

Thus, by using the exponential sum (hereafter, we will not specify that $\widehat{f}(\beta)$ is a finite sum)

$$
\widehat{f}(\beta) \stackrel{\text { def }}{=} \sum_{n \sim N} f(n) e(n \beta)
$$

we write

$$
\begin{aligned}
\mathscr{C}_{f}(h)= & \left.\sum_{\substack{m \sim N \\
n-m=h}} \sum_{\substack{n \sim N \\
n=h}} f(n) \overline{f(m)}+\|f\|_{\infty}^{2}|h|\right) \\
= & \int_{0}^{1}|\widehat{f}(\beta)|^{2} e(-h \beta) \mathrm{d} \beta+O\left(\|f\|_{\infty}^{2}|h|\right) \\
& \stackrel{(\mathrm{T})}{\sim} \int_{0}^{1}|\widehat{f}(\beta)|^{2} e(-h \beta) \mathrm{d} \beta .
\end{aligned}
$$

An appealing aspect is that the exponential sums, whose coefficients are correlations of a weight $w$, are nonnegative. More precisely,

$$
\begin{aligned}
\widehat{\mathscr{C}}_{w}(\beta) & =\sum_{h} \mathscr{C}_{w}(h) e(h \beta) \\
& =\sum_{h} \sum_{b-a=h} \sum_{w}(b) \overline{w(a)} e(h \beta)=\left|\sum_{n} w(n) e(n \beta)\right|^{2} \\
& =|\widehat{w}(\beta)|^{2}, \quad \forall \beta \in[0,1) .
\end{aligned}
$$

In particular, for the correlations of $u=\mathbf{1}_{[1, H]}$, one gets the well-known Fejér kernel

$$
\widehat{\mathscr{C}}_{u}(\beta)=|\widehat{u}(\beta)|^{2}=\left|\sum_{n \leq H} e(n \beta)\right|^{2} .
$$

More generally, the Fejér-Riesz theorem [16] states that every nonnegative exponential sum is the square modulus of another exponential sum. A particularly easy instance of this theorem follows by recalling that the Cesaro weight is the normalized correlation of $u$; that is, $C_{H}(h)=\mathscr{C}_{u}(h) / H$ (see Section 2). Hence, again Fejér's kernel makes its appearance in

$$
\begin{aligned}
\sum_{h} \max \left(1-\frac{|h|}{H}, 0\right) e(h \beta) & =\frac{1}{H} \sum_{h} \mathscr{C}_{u}(h) e(h \beta) \\
& =\frac{|\widehat{u}(\beta)|^{2}}{H} .
\end{aligned}
$$

We also say that the Cesaro weights are positive definite and think that this is an advantage, whereas the Selberg integral and the symmetry integral have weights $u$ and sgn that are far from being positive definite.

We expect to be able to exploit such a positivity condition, in the future.

\section{Essentially Bounded, Balanced, Quasi-Constant, and Stable Arithmetic Functions}

The wide class of arithmetic functions under our consideration consists of functions bounded asymptotically by every arbitrarily small power of the variable, in agreement with the definition (i.e., $f$ satisfies one of the Selberg Class axioms, the so-called Ramanujan hypothesis (see especially [15])),

$$
f \text { is essentially bounded } \stackrel{\text { def }}{\Longleftrightarrow} \forall \varepsilon>0 \quad f(n) \ll_{\varepsilon} n^{\varepsilon} \text {, }
$$

that we denote briefly by $f \lll 1$. A well-known prototype of an essentially bounded function is the divisor function $d_{k}$, whose Dirichlet series is $\zeta(s)^{k}$. Similarly, the Dirichlet series

$$
F(s) \stackrel{\text { def }}{=} \sum_{n=1}^{\infty} \frac{f(n)}{n^{s}}
$$

is defined in at least the right half-plane $\mathfrak{R}(s)>1$, whenever the generating function $f$ is essentially bounded. Assuming that $F$ is meromorphic, recall that the expansion of $F$ at $s=1$ leads to an asymptotic formula,

$$
\sum_{n \leq x} f(n)=\operatorname{Res}_{s=1} F(s) \frac{x^{s}}{s}+R_{f}(x) \stackrel{\text { def }}{=} x P_{f}(\log x)+R_{f}(x),
$$

defining $P_{f}$ as the residual polynomial of $f$, which either has degree (the polar order ord $_{s=1} F$ is the order of the pole of $F$ at $s=1$. In particular, $k$ is the polar order of $\left.\zeta^{k}\right) \operatorname{ord}_{s=1} F-1$ or vanishes identically when $\operatorname{ord}_{s=1} F<1$ (compare [15] for $F$ hypotheses). For the remainder term $R_{f}(x)$, a good estimate would be

$$
R_{f}(x) \lll x^{\alpha(f)}
$$

with a suitable $0 \leq \alpha(f)<1$ (negative values of $\alpha(f)$ are discarded as meaningless); see [15] formulas.

This is the case for any divisor function $d_{k}$. Indeed, from (*) of Section 1, one has

$$
\sum_{n \leq x} d_{k}(n)=x P_{k-1}(\log x)+\Delta_{k}(x) \quad \text { with } \Delta_{k}(x) \lll x^{\alpha_{k}},
$$

where the degree of the residual polynomial $P_{k-1}$ (see Section 1) is $k-1$, because the polar order of $\zeta^{k}$ is $k$. Here, $\alpha_{k} \leq 1-1 / k$ comes, inductively, from the elementary Dirichlet hyperbola method applied to $k=2$ (precisely, $\left.\Delta_{k}(x) \ll x^{1-1 / k} \log ^{k-2} x\right)$. We find, by partial summation, the logarithmic polynomial $p_{k-1}$ such that

$$
\sum_{n \leq x} p_{k-1}(\log n)=x P_{k-1}(\log x)+O\left(\log ^{k-1} x\right) .
$$

Thus, the balanced part of $d_{k}(n)$ is obtained by subtracting the very slowly increasing function $p_{k-1}(\log n)$ :

$$
\widetilde{d_{k}}(n) \stackrel{\text { def }}{=} d_{k}(n)-p_{k-1}(\log n) \text {. }
$$


Note that the product of $x^{s} / s$ and the Dirichlet series of $\widetilde{d_{k}}$ has zero residue at $s=1$.

Moreover, $(*)$ is equivalent to

$$
\sum_{n \leq x} \widetilde{d_{k}}(n) \lll x^{\alpha_{k}} .
$$

This invites us to formulate the following definitions (although with a different meaning, such a terminology has been coined by Ben Green and Terence Tao. Mainly, Green [17] calls a function $f-\delta$ balanced when $f$ is a characteristic function of a set with density $\delta$ ):

$$
\begin{aligned}
f \text { is balanced } \stackrel{\text { def }}{\Longleftrightarrow} \operatorname{Res}_{s=1} \frac{x^{s}}{s} F(s) & =\operatorname{Res}_{s=1} \frac{x^{s}}{s} \sum_{n=1}^{\infty} \frac{f(n)}{n^{s}} \\
& =0, \quad \forall x
\end{aligned}
$$

(i.e., $F$ has an analytic continuation in $s=1$ ),

$f$ is well-balanced of exponent $\alpha$

$$
\stackrel{\text { def }}{\Longleftrightarrow} \sum_{n \leq x} f(n) \lll x^{\alpha} \quad \text { for some } \alpha \in[0,1) \text {. }
$$

Of course, well-balanced implies balanced (because, from previous bound, $F(s)$ is regular at $s=1$ ). However, the converse needs not to be true; particularly, $\Lambda(n)-1$ is a balanced function (by the prime number Theorem), but the existence of an exponent $\alpha<1$ is far from being proved (compare [18]).

An essentially bounded arithmetic function $a: \mathbb{N} \rightarrow \mathbb{C}$ is said to be quasi-constant if there exists $A \in C^{1}([1,+\infty), \mathbb{C})$ such that (the condition on the derivative $A^{\prime}$ implies that $A$ is essentially bounded, provided that $a$ depends only on $n$. However, we do not exclude the possibility that $a$ and $A$ might depend on auxiliary parameters) $A^{\prime}(t) \lll 1 / t$ and $A=a$ on $\mathbb{N}$. Clearly, the logarithmic polynomial $p_{k-1}(\log n)$ is quasi-constant with respect to $n$. This, together with the fact that $\widetilde{d_{k}}$ is a well-balanced arithmetic function of exponent $\alpha_{k}$, suggests the following further definition.

An arithmetic function $f$ is stable of exponent $\alpha$ if there exist a quasi-constant function $a$ and a well-balanced function $b$ of exponent $\alpha$ such that $f=a+b$, whereas the amplitude of $f$ is defined as

$$
\alpha(f) \stackrel{\text { def }}{=} \inf \{\alpha \in(0,1): f \text { is stable of exponent } \alpha\} .
$$

In what follows, we will assume that stable $f$ have a logarithmic polynomial (above is the general definition).

Recall that the Dirichlet divisor problem requires to prove the conjectured amplitude $\alpha_{2}=\alpha(\mathbf{d})=1 / 4$, while one infers $\alpha_{2} \leq 1 / 2$ by the Dirichlet hyperbola method and the best known bound at the present moment is $\alpha_{2} \leq 141 / 416$ (Huxley, 2003). In the sequel, $\alpha_{k}=\alpha\left(d_{k}\right)$ is the best possible exponent in $(*)$ and $(\widetilde{*})$.

According to Ivić [3], the mean value in the Selberg integral of an arithmetic function $f$, with Dirichlet series $F(s)$ converging absolutely (at least) in $\mathfrak{R}(s)>1$ and meromorphic in $\mathbb{C}$, has analytic form given by

$$
M_{f}(x, H) \stackrel{\text { def }}{=} H\left(P_{f}(\log x)+P_{f}^{\prime}(\log x)\right),
$$

where $P_{f}^{\prime}$ is the derivative of the residual polynomial $P_{f}$. We remark that $M_{f}(x, H)$ is linear in $f$ and is separable; that is, the variables $H, x$ are separated. The logarithmic polynomial $p_{f} \stackrel{\text { def }}{=} P_{f}+P_{f}^{\prime}$ has the property

$$
\begin{aligned}
p_{f}(\log x) & =\operatorname{Res}_{s=1} F(s) \frac{x^{s-1}}{s}+\operatorname{Res}_{s=1} F(s) \frac{(s-1) x^{s-1}}{s} \\
& =\operatorname{Res}_{s=1} F(s) x^{s-1} .
\end{aligned}
$$

For $f=d_{k}$, this identity permits us to compare $[5,6]$ results with ours (see Section 1).

In particular, the analytic form of the mean value in the Selberg integral of $d_{3}$ is explicitly given by

$$
\begin{aligned}
M_{3}(x, H) & =H\left(P_{2}(\log x)+P_{2}^{\prime}(\log x)\right) \\
& =H\left(\frac{1}{2} \log ^{2} x+3 \gamma \log x+3 \gamma^{2}+3 \gamma_{1}\right),
\end{aligned}
$$

with $P_{2}(t) \stackrel{\text { def }}{=} t^{2} / 2+(3 \gamma-1) t+\left(3 \gamma^{2}+3 \gamma_{1}-3 \gamma+1\right)$, where $\gamma$ is the Euler-Mascheroni constant and

$$
\gamma_{1} \stackrel{\text { def }}{=} \lim _{m}\left(\frac{\log ^{2} m}{2}-\sum_{j \leq m} \frac{\log j}{j}\right) .
$$

Regarding the (short) sum of $d_{3}$ weighted with Cesaro weights, that is,

$$
\sum_{0 \leq|n-x| \leq H}\left(1-\frac{|n-x|}{H}\right) d_{3}(n),
$$

Proposition 16 in Section 7 (see Remark 17 too) suggests (from Proposition 14 in Section 7) that the expected mean value is

$$
\begin{aligned}
\widetilde{M}_{3}(x, H) \stackrel{\text { def }}{=} H\left(\sum_{q \leq x / N_{3}} \frac{\mathbf{d}(q)}{q}+\sum_{d_{1}<N_{3}} \frac{1}{d_{1}} \sum_{d_{2} \leq x / d_{1} N_{3}} \frac{1}{d_{2}}\right. \\
\left.+\left(\sum_{d<N_{3}} \frac{1}{d}\right)^{2}\right),
\end{aligned}
$$

where $N_{3}=\left[N^{1 / 3}\right]$. Next, Proposition 9 justifies the interchange of analytic form $M_{3}(x, H)$ and arithmetic form of the mean value $\widetilde{M}_{3}(x, H)$, inside $\widetilde{J}_{3}(N, H)$; its proof is designed for the specific case $k=3$.

Proposition 9. Uniformly, for every $x \sim N$, one has

$$
\widetilde{M}_{3}(x, H)-M_{3}(x, H) \lll H N^{-1 / 3} .
$$

The idea of the proof (details on arxiv: v3 of this paper) is to apply Amitsur's formula [19] with Tull's error term [20] (Amitsur derived a symbolic method to calculate main terms of asymptotic formulas. Tull gave a refined partial summation that allows us to transfer the error terms from the formula 
for the sum $\sum_{q \leq Q} \mathbf{d}(q)$, like Dirichlet's classical $O(\sqrt{Q})$, to this formula); that is,

$$
\sum_{q \leq Q} \frac{\mathbf{d}(q)}{q}=\frac{\log ^{2} Q}{2}+2 \gamma \log Q+\left(\gamma^{2}+2 \gamma_{1}\right)+O\left(\frac{1}{\sqrt{Q}}\right) .
$$

Finally, by Lemma 7, the Selberg integrals and the modified one for a real and essentially bounded function $f$ are related to the correlation averages (see Remark 8), respectively, as

$$
\begin{aligned}
& J_{f}(N, H) \stackrel{(\mathrm{T})}{\sim} \sum_{h} \mathscr{C}_{u}(h) \mathscr{C}_{f}(h) \\
&-2 \sum_{n} f(n) \sum_{x \sim N} u(n-x) M_{f}(x, H) \\
&+\sum_{x \sim N} M_{f}(x, H)^{2}, \\
& \widetilde{J}_{f}(N, H) \stackrel{(\mathrm{T})}{\sim} \sum_{h} \mathscr{C}_{\mathscr{C}_{u} / H}(h) \mathscr{C}_{f}(h) \\
& \\
&-2 \sum_{n} f(n) \sum_{x \sim N} \frac{\mathscr{C}_{u}(n-x)}{H} M_{f}(x, H) \\
&+\sum_{x \sim N} M_{f}(x, H)^{2} .
\end{aligned}
$$

In particular, when $f$ is also balanced, that is, $M_{f}(x, H)$ vanishes identically, from these formulas, we get

$$
\begin{gathered}
J_{f}(N, H) \stackrel{(\mathrm{T})}{\sim} \sum_{h} \mathscr{C}_{u}(h) \mathscr{C}_{f}(h), \\
\widetilde{J}_{f}(N, H) \stackrel{(\mathrm{T})}{\sim} \sum_{h} \mathscr{C}_{\mathscr{C}_{u} / H}(h) \mathscr{C}_{f}(h) .
\end{gathered}
$$

\section{Averages of Correlations: \\ Smoothing Correlations' Formulas by Arithmetic Means}

Recalling that

$$
\mathscr{C}_{f}(h) \stackrel{(\mathrm{T})}{\sim} \int_{0}^{1}|\widehat{f}(\beta)|^{2} e(-h \beta) \mathrm{d} \beta
$$

with $\widehat{f}(\beta)=\sum_{n \sim N} f(n) e(n \beta)$, one easily infers the formulas

$$
\begin{gathered}
\sum_{h} u(h) \mathscr{C}_{f}(h)=\int_{0}^{1}|\widehat{f}(\beta)|^{2} \widehat{u}(-\beta) \mathrm{d} \beta+O\left(H^{2}\|f\|_{\infty}^{2}\right) \\
\stackrel{(\mathrm{T})}{\sim} \int_{0}^{1}|\widehat{f}(\beta)|^{2} \widehat{u}(-\beta) \mathrm{d} \beta \\
\sum_{h} \mathscr{C}_{u}(h) \mathscr{C}_{f}(h)=\int_{0}^{1}|\widehat{f}(\beta)|^{2} \widehat{\mathscr{C}}_{u}(-\beta) \mathrm{d} \beta \\
+O\left(H^{3}\|f\|_{\infty}^{2}\right) \\
\stackrel{(\mathrm{T})}{\sim} \int_{0}^{1}|\widehat{f}(\beta)|^{2}|\widehat{u}(\beta)|^{2} \mathrm{~d} \beta, \\
\sum_{h} \mathscr{C}_{\mathscr{C}_{u} / H}(h) \mathscr{C}_{f}(h)=\int_{0}^{1}|\widehat{f}(\beta)|^{2} \frac{\left|\widehat{\mathscr{C}}_{u}(\beta)\right|^{2}}{H^{2}} \mathrm{~d} \beta \\
+O\left(H^{3}\|f\|_{\infty}^{2}\right) \\
\stackrel{(\mathrm{T})}{\sim} \int_{0}^{1}|\widehat{f}(\beta)|^{2}|\widehat{u}(\beta)|^{2} \cdot \frac{|\widehat{u}(\beta)|^{2}}{H^{2}} \mathrm{~d} \beta .
\end{gathered}
$$

In particular, for every balanced real function $f \lll 1$, from the last formulas of the previous section, we get

$$
\begin{aligned}
& J_{f}(N, H) \stackrel{(\mathrm{T})}{\sim} \int_{0}^{1}|\widehat{f}(\beta)|^{2}|\widehat{u}(\beta)|^{2} \mathrm{~d} \beta, \\
& \widetilde{J}_{f}(N, H) \stackrel{(\mathrm{T})}{\sim} \int_{0}^{1}|\widehat{f}(\beta)|^{2} \frac{|\widehat{u}(\beta)|^{4}}{H^{2}} \mathrm{~d} \beta .
\end{aligned}
$$

Formulas (I), (II), and (III) correspond, respectively, to the following iterations of correlations' averages.

1st generation:

$$
\sum_{h \leq H} \mathscr{C}_{f}(h)=\sum_{h} u(h) \mathscr{C}_{f}(h) \quad \text { (sums of correlations), }
$$

2nd generation:

$\sum_{h \leq H} \sum_{0 \leq|a|<h} \mathscr{C}_{f}(a)=\sum_{h} \mathscr{C}_{u}(h) \mathscr{C}_{f}(h) \quad$ (double sums),

3rd generation:

$$
\sum_{h} \sum_{h_{2}-h_{1}=h} \frac{\mathscr{C}_{u}\left(h_{1}\right) \mathscr{C}_{u}\left(h_{2}\right)}{H^{2}} \mathscr{C}_{f}(h)=\sum_{h} \mathscr{C}_{\mathscr{C}_{u} / H}(h) \mathscr{C}_{f}(h)
$$

(average of double sums).

Such an obstinate process of averaging is motivated by the fact that it is rarely possible to prove an asymptotic formula 
for the single correlation $\mathscr{C}_{f}$. This counts the number of $h$ twins (see Section 3) not only when $f$ is a pure characteristic function (the von Mangoldt function is a typical case). In general, the underlying Diophantine equation is a binary problem still out of reach; see Section 3 of [7] (and also http://arxiv.org/: v3 of present paper). On the other hand, for higher generations of correlations' averages, we get smoother averages; for which, consequently, there is more hope of proving nontrivial estimates. However, even for the 2nd generation, this hope is quite frustrated by the lack of efficient elementary methods to bound the Selberg integral directly. Indeed, Ivić [3] applies the $2 k$ th moments of $\zeta$, since $J_{k}$ has a strong connection with them (see Section 8).

Further, it is interesting to analyze the cost of the loss when nontrivial information on the correlations' averages at some $n$th generation level is transferred to the $(n-1)$ th generation (see Lemmas 10 and 12).

In this regard, if $f$ is real, essentially bounded, and balanced, then the trivial inequality $\widehat{u} \ll H$ gives

$$
\widetilde{J}_{f}(N, H) \lll J_{f}(N, H)+H^{3} .
$$

Then, in order to obtain an inequality in the opposite direction, we prove the following lemma.

Lemma 10. For every essentially bounded, balanced, and real arithmetic function $f$ and for every $H=o(N)$,

$$
J_{f}(N, H) \lll N^{1 / 2} H\left(\widetilde{J}_{f}(N, H)^{1 / 2}+H^{3 / 2}\right) .
$$

Proof. By applying Cauchy's inequality and Parseval's identity, we see that

$$
\begin{aligned}
& \int_{0}^{1}|\widehat{f}(\beta)|^{2}|\widehat{u}(\beta)|^{2} \mathrm{~d} \beta \\
& \quad \ll \sqrt{\sum_{n \sim N}|f(n)|^{2} \int_{0}^{1}|\widehat{f}(\beta)|^{2}|\widehat{u}(\beta)|^{4} \mathrm{~d} \beta} \\
& \ll \sqrt{N \int_{0}^{1}|\widehat{f}(\beta)|^{2}|\widehat{u}(\beta)|^{4} \mathrm{~d} \beta .}
\end{aligned}
$$

The Lemma is proved (using $H^{3} \ll N^{1 / 2} H^{5 / 2}$ ), because the formulas for $J_{f}(N, H)$ and $\widetilde{J}_{f}(N, H)$ from (II) and (III) yield

$$
\begin{gathered}
J_{f}(N, H) \lll \int_{0}^{1}|\widehat{f}(\beta)|^{2}|\widehat{u}(\beta)|^{2} \mathrm{~d} \beta+H^{3}, \\
\int_{0}^{1}|\widehat{f}(\beta)|^{2}|\widehat{u}(\beta)|^{4} \mathrm{~d} \beta \lll H^{2}\left(\widetilde{J}_{f}(N, H)+H^{3}\right) .
\end{gathered}
$$

Now, let us prove Theorems 1 and 2.

Proof of Theorems 1 and 2. First, we note that $M_{k}(x, H) \sim$ $\sum_{x<n \leq x+H} p_{k-1}(\log n)$, where $p_{k-1}$ is the logarithmic polynomial of $d_{k}$ and the implicit remainders give a negligible contribution in view of the stated estimate for $J_{k}(N, H)$. Then, from Lemma 10, it follows that if, for some $H$, a nontrivial estimate of the form

$$
\widetilde{J}_{f}(N, H) \ll \frac{N H^{2}}{G}
$$

holds with some gain $G \rightarrow \infty$, then, for the same range of $H$, one has

$$
J_{f}(N, H) \lll N H^{2} G^{-1 / 2}+N^{1 / 2} H^{5 / 2} .
$$

Thus, using these inequalities for the balanced part of $d_{k}(n)$, that is, $f(n)=\widetilde{d_{k}}(n)=d_{k}(n)-p_{k-1}(\log n)$, Theorems 1 and 2 follow by taking, respectively, $G=H N^{-\varepsilon}$ in the Conjecture CL for $k=3$ and $G=N^{4 \varepsilon_{1}}$ in the General Conjecture CL for $k=4,5,6,7$.

Remark 11. Lemma 10 reveals that, applying only Cauchy inequality, a third generation gain $G \ll N / H$ leads to the gain $\sqrt{G}$ for the second generation. We say that the exponent gain has halved.

What about the trade of information from the second generation to the first one?

Let us define the deviation of a stable arithmetic function $f$, with $\operatorname{logarithmic}$ polynomial $a(n)=p_{f}(\log n)$, as

$$
\mathbb{D}_{f}(N, H) \stackrel{\text { def }}{=} \sum_{h \leq H} \mathscr{C}_{f}(h)-H \sum_{x \sim N} a(x)^{2} .
$$

Lemma 12 estimates $\mathbb{D}_{f}(N, H)$ in terms of the Selberg integral of $f$ (when $f \lll 1$ is real and stable).

Lemma 12. Let $f$ be an essentially bounded, stable, and real arithmetic function with amplitude $\alpha=\alpha(f)$. Then, for every $H=o(N)$, one has

$$
\mathbb{D}_{f}(N, H) \lll N^{1 / 2} J_{f}(N, H)^{1 / 2}+N^{\alpha} H+H^{2} .
$$

Proof. Let $f=a+b$ where $a(n)=p_{f}(\log n)$ as above and let $b$ be well-balanced. Then, we write

$$
\begin{aligned}
\sum_{h \leq H} \mathscr{C}_{f}(h) \stackrel{(\mathrm{T})}{\sim} & \sum_{h \leq H} \sum_{n \sim N} f(n) f(n+h) \\
= & \sum_{x \sim N} f(x) \sum_{x<m \leq x+H} f(m) \\
= & \sum_{x \sim N} f(x) \sum_{x<m \leq x+H} a(m) \\
& +\sum_{x \sim N} f(x) \sum_{x<m \leq x+H} b(m) .
\end{aligned}
$$

By the Cauchy inequality, one has

$$
\begin{aligned}
& \sum_{x \sim N} f(x) \sum_{x<m \leq x+H} b(m) \\
& \ll\left(\sum_{x \sim N}|f(x)|^{2} \sum_{x \sim N}\left|\sum_{x<n \leq x+H} f(n)-M_{f}(x, H)\right|^{2}\right)^{1 / 2} \\
& \ll N^{1 / 2} J_{f}(N, H)^{1 / 2},
\end{aligned}
$$


where the mean value is (see Section 2) $M_{f}(x, H) \stackrel{\text { def }}{=}$ $\sum_{x<n \leq x+H} a(n) \stackrel{(\mathrm{T})}{\sim} a(x) H$, since $a$ is quasi-constant. Hence, we get

$$
\begin{aligned}
\sum_{h \leq H} \mathscr{C}_{f}(h)= & H \sum_{x \sim N} a(x)^{2}+H \sum_{x \sim N} a(x) b(x) \\
& +O_{\varepsilon}\left(N^{1 / 2+\varepsilon} J_{f}(N, H)^{1 / 2}+N^{\varepsilon} H^{2}\right)
\end{aligned}
$$

and the lemma follows applying partial summation to $\sum_{x \sim N} a(x) b(x)$, since $f$ is stable with amplitude $\alpha$.

Remark 13. From previous Lemma, if, for a suitable $G=$ $o(H), G \rightarrow \infty$, one has

$$
J_{f}(N, H) \ll N H^{2} G^{-1 / 2} ;
$$

then

$$
\mathbb{D}_{f}(N, H) \lll N H G^{-1 / 4}+N^{\alpha} H+H^{2} .
$$

In particular, for $f=d_{k}$, we get (the essence of Corollary 5; see above)

$$
\begin{aligned}
& J_{k}(N, H) \ll_{\varepsilon} N^{1-3 \varepsilon} H^{2} \\
& \quad \Longrightarrow \mathbb{D}_{k}(N, H) \ll_{\varepsilon} N^{1-\varepsilon} H+N^{\varepsilon}\left(N^{\alpha_{k}} H+H^{2}\right) .
\end{aligned}
$$

In conclusion, Lemmas 10 and 12 provide the following chain of implications of nontrivial bounds:

$$
\begin{aligned}
\widetilde{J}_{f}(N, H) & \lll N H^{2} G^{-1} \Longrightarrow J_{f}(N, H) \\
& \lll N H^{2} G^{-1 / 2} \Longrightarrow \mathbb{D}_{f}(N, H) \lll N H G^{-1 / 4}
\end{aligned}
$$

The exponent gains halves at each step. If it is a neat positive one, we say that $f$ is stable through generations.

In the future, we will explore the hard case of stable $f$ (possibly) having no logarithmic polynomial.

\section{Asymptotic Formulas for $d_{3}$ in Almost All Short Intervals}

We turn our attention to $J_{3}$ nontrivial bounds, postponing those on $J_{k}$ to the next section. Ivić [3] proved that

$$
J_{3}(N, H) \ll N^{1-\varepsilon_{1}} H^{2}
$$

for the width $\theta>1 / 6$, with a neat exponent gain $\varepsilon_{1}>0$. In other words, if $\theta>\theta_{3}$ is the width range for such an inequality to hold, Ivić has proved $\theta_{3}=1 / 6$.

This comes from $\sigma_{3} \leq 7 / 12$, where $\sigma_{k}$ is the Carlson's abscissa for the Riemann zeta $2 k$ th moment:

$$
\sigma_{k} \stackrel{\text { def }}{=} \inf \left\{\sigma \in[0,1]: \int_{1}^{T}|\zeta(\sigma+i t)|^{2 k} \mathrm{~d} t \ll T\right\}
$$

(see [4] to get some of the known upper bounds for $\sigma_{k}$ ).
Conjecture CL provides improvements on Ivićs result, since, for every width $\theta \leq 1 / 3$, it yields the best possible estimate, that is, the square-root cancellation $\widetilde{J}_{3}(N, H) \lll N H$ (compare with the abovementioned lower bound $J_{3}(N, H) \gg$ $N H \log ^{4} N$ holding for $0<\theta<1 / 3$; see [2]), which in turn implies the optimal $\theta_{3}=0$ through the arguments of Section 5 (compare Lemma 10 and Theorems 1 and 2 proofs).

Our estimate for $J_{3}$ leads to improvements on $[5,6]$ for $d_{3}$ deviation. That is what we prove now.

Proof of Corollary 3. From the decomposition $d_{3}(n)=$ $p_{2}(\log n)+\widetilde{d_{3}}(n)$ introduced in Section 4 , one gets

$$
\begin{aligned}
\mathscr{C}_{3}(h) \stackrel{\text { def }}{=} & \sum_{n \sim N} d_{3}(n) d_{3}(n-h)=\sum_{N+h<m \leq 2 N+h} d_{3}(m+h) d_{3}(m) \\
= & \sum_{n \sim N} d_{3}(n+h) d_{3}(n)+O_{\varepsilon}\left(N^{\varepsilon}|h|\right) \\
= & \sum_{n \sim N} \widetilde{d_{3}}(n) \widetilde{d_{3}}(n+h)+2 \sum_{n \sim N} \widetilde{d_{3}}(n) p_{2}(\log n) \\
& +\sum_{n \sim N} p_{2}(\log n)^{2}+O_{\varepsilon}\left(N^{\varepsilon}|h|\right),
\end{aligned}
$$

where we recall that $d_{3}, \widetilde{d_{3}}$ are essentially bounded and $p_{2}(\log n)$ is a quasi-constant function of $n$. Therefore,

$$
\begin{aligned}
\mathbb{D}_{3}(N, H)= & \sum_{h \leq H} \widetilde{\mathscr{C}_{3}}(h)-H \sum_{n \sim N} p_{2}(\log n)^{2} \\
= & \sum_{n \sim N} \widetilde{d_{3}}(n) \sum_{n<m \leq n+H} \widetilde{d_{3}}(m) \\
& +2 H \sum_{n \sim N} \widetilde{d_{3}}(n) p_{2}(\log n)+O_{\varepsilon}\left(N^{\varepsilon} H^{2}\right) .
\end{aligned}
$$

By applying partial summation and $(\widetilde{*})$, one has

$$
\begin{aligned}
\sum_{n \sim N} \widetilde{d_{3}}(n) p_{2}(\log n)= & p_{2}(\log (2 N)) \sum_{n \sim N} \widetilde{d_{3}}(n) \\
& -\int_{N}^{2 N} \sum_{N<n \leq t} \widetilde{d_{3}}(n) \frac{p_{2}^{\prime}(\log t)}{t} \mathrm{~d} t \\
& \lll \max _{t \leq 2 N}\left|\sum_{N<n \leq t} \widetilde{d_{3}}(n)\right| \lll N^{\alpha_{3}} .
\end{aligned}
$$

Since $J_{3}(N, H) \gg N H L^{4}$ holds for at least width $0<\theta<$ $1 / 3$ (see [2] and recall that $L=\log N$ ), the conclusion from Theorem 1 follows, because Cauchy's inequality implies

$$
\begin{aligned}
& \sum_{n \sim N} \widetilde{d_{3}}(n) \sum_{n<m \leq n+H} \widetilde{d_{3}}(m) \\
& \quad \lll \sqrt{N\left(\sum_{x \sim N}\left|\sum_{x<m \leq x+H} d_{3}(m)-M_{3}(x, H)\right|^{2}+\frac{H^{4} L^{2}}{N}\right)} \\
& \lll \sqrt{N J_{3}(N, H)},
\end{aligned}
$$


where (compare Section 2)

$$
\begin{aligned}
M_{3}(x, H) & =H p_{2}(\log x) \\
& =\sum_{x<m \leq x+H} p_{2}(\log m)+O\left(x^{-1} H^{2} L\right) .
\end{aligned}
$$

\section{Main Theme: From All Long Intervals to Almost All Short Intervals}

The inductive identity $d_{k}=d_{k-1} * \mathbf{1}$ invites us to explore the possibility to infer formulas for $d_{k}$ in almost all short intervals, from suitable information on $d_{k-1}$ in long intervals.

However, the known bounds on the amplitudes $\alpha_{k}$ seem to be a first serious bottle-neck. Further, while it might be comparatively easy to attack Conjecture CL, the path climbs up drastically when $k \in\{4,5,6,7\}$. Although a general $k$ folding method is available (Proposition 14, the core of our elementary approach), we want to prove the following meansquare proximity of analytic and arithmetic forms of s.i. mean value:

$$
\sum_{x \sim N}\left|M_{k}(x, H)-\widetilde{M}_{k}(x, H)\right|^{2} \lll N^{1-2 / k} H^{2}
$$

for a suitable arithmetic mean value $\widetilde{M}_{k}(x, H)$. The right form is suggested by Proposition 14 that also implies (via the Large Sieve inequality) a nontrivial bound for $J_{w, d_{k}}(N, H)$ in short intervals of width $\theta>1-2 / k$ (for any $w$ satisfying a property shared by our weights $u, C_{H}$, and sgn), indeed, Proposition 16.

On the other hand, the above mean-square proximity of $M_{k}(x, H)$ and $\widetilde{M}_{k}(x, H)$, at the present moment, is obtained with a gain weaker than $N^{2 / k}$; see next Proposition 18, where we apply the nontrivial estimates for $J_{k}(N, H)$ given by Ivić [3] in the common range where Proposition 16 also gives nontrivial bounds.

Now, for the so-called $k$-folding method, we need some ad hoc notation. For all $k \geq 2$, let us consider

$$
\begin{aligned}
S\left(x ; a_{k}, w\right) & \stackrel{\text { def }}{=} \sum_{n} a_{k}(n) w(n-x) \\
& =\sum_{n_{1}} \cdots \sum_{n_{k}} a\left(n_{1}\right) \cdots a\left(n_{k}\right) w\left(n_{1} \cdots n_{k}-x\right),
\end{aligned}
$$

where $a_{k} \stackrel{\text { def }}{=} \underbrace{a * \cdots * a}_{k \text { times }}$ is the $k$-fold Dirichlet product of a fixed arithmetic function $a$ and the weight $w:[-H, H] \rightarrow \mathbb{C}$ is uniformly bounded with respect to $H$, independent of $x$. Set $N_{k} \stackrel{\text { def }}{=}\left[N^{1 / k}\right]$ and denote

$$
g_{k}(q)=g_{k}\left(q, a, N_{k}\right) \stackrel{\text { def }}{=} a_{k-1}(q)+\sum_{j \leq k-1} a_{k-1}^{(j)}(q)
$$

where

$$
a_{k-1}^{(j)}(q) \stackrel{\text { def }}{=} \sum_{\substack{n_{1} \\ n_{1} \cdots n_{k-1}=q \\ n_{1}, \cdots, n_{j}<N_{k}}} \ldots \sum_{\substack{n_{k-1} \\ n_{1}}} a\left(n_{1}\right) \cdots a\left(n_{k-1}\right), \quad \forall j \leq k-1 .
$$

Proposition 14. If $a: \mathbb{N} \rightarrow \mathbb{C}$ is essentially bounded, then uniformly, for $x \in[N+H, 2 N]$, one has

$$
\begin{aligned}
S\left(x ; a_{k}, w\right)= & \sum_{q \leq x / N_{k}} g_{k}(q) \\
& \times \sum_{\substack{0 \leq|n-x| \leq H \\
n=0(\bmod q)}} a\left(\frac{n}{q}\right) w(n-x) \\
& +O_{k, \varepsilon}\left(N^{\varepsilon}\left(\frac{H}{N^{1 / k}}+\frac{H^{2}}{N}+1\right)\right) .
\end{aligned}
$$

The proof runs on the lines of (initial part of) the proof of Corollary 1 in [2] and we will omit it.

Remark 15. The uncovered range $N<x<N+H$ gives in mean-square a tail contribution $\lll H^{3}$.

We keep hereafter the notation $g_{k}(q)$ even for $a=1$ and set $S_{k}(x, w) \stackrel{\text { def }}{=} S\left(x ; d_{k}, w\right)=\sum_{n} d_{k}(n) w(n-x)$.

Let us state the following consequence of Proposition 14, denoting $\widehat{w}(\beta) \stackrel{\text { def }}{=} \sum_{0 \leq|h| \leq H} w(h) e(h \beta)$.

Proposition 16. Assume that $w$ is uniformly bounded in $[-H, H]$ and

$$
\frac{1}{q^{2}} \sum_{\substack{j \leq q \\(j, q)=1}}\left|\widehat{w}\left(\frac{j}{q}\right)\right|^{2} \lll \min \left(1, \frac{H}{q}\right) .
$$

Then, for every integer $k>2$, one has

$$
\begin{aligned}
J_{w, d_{k}}(N, H) \stackrel{\text { def }}{=} \sum_{x \sim N}\left|S_{k}(x, w)-\widehat{w}(0) \sum_{q \leq x / N_{k}} \frac{g_{k}(q)}{q}\right|^{2} \\
\\
\lll N^{2-2 / k} H+H^{3} .
\end{aligned}
$$

The proof applies the Large Sieve inequality in the form given by Lemma 3 of [13] and will appear elsewhere.

Remark 17. The Cesaro weight $C_{H}$ satisfies previous assumptions: recalling Section 3 last formula and the well-known 
$|\widehat{u}(\beta)| \ll \min \left(H,\|\beta\|^{-1}\right)$, if we set $H_{q} \stackrel{\text { def }}{=} q\{H / q\}$, then (for $H_{q}>0$; otherwise, the following vanishes)

$$
\begin{aligned}
\frac{1}{q^{2}} \sum_{\substack{j \leq q \\
(j, q)=1}}\left|\widehat{C_{H}}\left(\frac{j}{q}\right)\right|^{2} & =\frac{1}{q^{2} H^{2}} \sum_{\substack{j \leq q \\
(j, q)=1}}\left|\sum_{n \leq H_{q}} e_{q}(n j)\right|^{4} \\
& \ll \frac{1}{q^{2} H^{2}} \sum_{\substack{|j| \leq q / 2 \\
(j, q)=1}} \min \left(H_{q}, \frac{1}{\|j / q\|}\right)^{4} \\
& \ll \frac{1}{q^{2} H^{2}} \sum_{j \leq q / 2} \min \left(H_{q}, \frac{q}{j}\right)^{4} \\
& \ll \frac{H_{q}^{4}}{q^{2} H^{2}} \sum_{j \leq q / H_{q}} 1+\frac{q^{2}}{H^{2}} \sum_{j>q / H_{q}} \frac{1}{j^{4}} \\
& \ll \frac{H_{q}^{3}}{q H^{2}} \ll \min \left(1, \frac{H}{q}\right) .
\end{aligned}
$$

Similar calculations prove that $w=u$ and $w=$ sgn satisfy previous Proposition assumptions, too.

The replacement, in general, of analytic $M_{f}$ by arithmetic $\widetilde{M}_{f}$ lies at the heart of our arithmetic approach.

We see that the analytic form $M_{k}(x, w)=\widehat{w}(0) p_{k-1}(\log x)$ is close to the arithmetic form in Proposition 16:

$$
\widetilde{M}_{k}(x, w) \stackrel{\text { def }}{=} \widehat{w}(0) \sum_{q \leq x / N_{k}} \frac{g_{k}(q)}{q} .
$$

Proposition 18. For every integer $k \geq 3$, there exists $G=$ $G(k)>0$ such that

$$
\sum_{x \sim N}\left|\sum_{q \leq x / N_{k}} \frac{g_{k}(q)}{q}-p_{k-1}(\log x)\right|^{2} \ll N^{1-G}
$$

The proof follows by comparing (above quoted) Ivićs bounds with Proposition 16 and will appear elsewhere.

Notice that, for $k=3$, this Proposition yields a much weaker inequality than Proposition 9.

Remark 19. As for the case $k=3$, implications in Section 5 and Propositions 16 and 18 immediately prove Corollary 4.

\section{Conditional Bounds for the Moments of $\zeta$ on the Critical Line}

We do not use explicitly any deep property of $\zeta$, relying upon $\alpha_{k}$ known bounds, but these, actually, follow from nontrivial $I_{k}(T)$ estimates (for a clear digression on this; see the wonderful book [4] by Ivić).

On the other hand, bounds for $J_{k}$ have nontrivial consequences on $I_{k}(T)$, as applied in next proof.
Proof of Theorem 6. For $T \rightarrow \infty$ and for every $\varepsilon>0$, Theorem 1.1 of [7] yields (« saves $O_{\varepsilon}\left(T^{\mathcal{E}}\right)$ here)

$$
I_{k}(T) \lll T+T \max _{T^{1+\varepsilon} \ll N \ll T^{k / 2}} T N^{-2} \max _{0<H \ll N / T} J_{k}(N, H) .
$$

Indeed, in Theorem 1.1 of [7], one finds the same inequality, with $J_{k}(N, H)$ replaced by the Selberg integral

$$
\int_{H x^{\varepsilon}}^{x}\left|\sum_{t<n \leq t+H} d_{k}(n)-M_{k}(t, H)\right|^{2} \mathrm{~d} t .
$$

However, an easy dyadic argument allows one to replace $\left[H x^{\varepsilon}, x\right]$ by an interval like $[N, 2 N]$, while the substitution of the integral on $[N, 2 N]$ with $J_{k}(N, H)$ generates only negligible remainder terms.

Since $J_{k}(N, H) \lll N^{1+A} H^{1+B}$ holds for $H \ll N^{1-2 / k}$, by hypothesis, the previous inequality implies

$$
\begin{aligned}
I_{k}(T) & \lll T+T \max _{T^{1+\varepsilon} \ll N \ll T^{k / 2}} N^{A}\left(\frac{N}{T}\right)^{B} \\
& \lll T+T\left(T^{k / 2}\right)^{A}\left(T^{k / 2-1}\right)^{B} \\
& \lll T^{1+(k / 2)(A+B)-B} .
\end{aligned}
$$

Remark 20. Define the excess $E_{k}$ to be such that $I_{k}(T) \lll$ $T^{1+E_{k}}$; Theorem 6 yields $E_{k}=(k / 2)(A+B)-B$. Known values are $E_{3}=1 / 4, E_{4}=1 / 2, E_{5}=3 / 4$ followed by Hölder's inequality from $E_{2}=0$ and $E_{6}=1$ (see Section 2 in [7]).

According to Section 5, our $E_{k}$ belong to a 2 nd generation approach, while Ivić's [8] is a 1st generation one. Under Conjecture CL, our new approach, based on a modified version of Gallagher's Lemma [21], gives $E_{3}=0$.

\section{Conflict of Interests}

The authors declare that there is no conflict of interests regarding the publication of this paper.

\section{Acknowledgments}

The authors are very grateful to Alberto Perelli for interesting discussions and invaluable suggestions. The authors wish to give Corollary 5 as a gift to Professor Aleksandar Ivić.

\section{References}

[1] A. Selberg, "On the normal density of primes in small intervals, and the difference between consecutive primes," Archiv for Mathematik og Naturvidenskab, vol. 47, no. 6, pp. 87-105, 1943.

[2] G. Coppola, "On some lower bounds of some symmetry integrals," Afrika Matematika, vol. 25, no. 1, pp. 183-195, 2014.

[3] A. Ivić, "On the mean square of the divisor function in short intervals," Journal de Théorie des Nombres de Bordeaux, vol. 21, no. 2, pp. 251-261, 2009. 
[4] A. Ivić, The Riemann Zeta-Function, John Wiley \& Sons, New York, NY, USA, 1985, 2nd edition, Dover, Mineola, NY, USA, 2003.

[5] S. Baier, T. D. Browning, G. Marasingha, and L. Zhao, "Averages of shifted convolutions of $d_{3}(n)$," Proceedings of the Edinburgh Mathematical Society. Series II, vol. 55, no. 3, pp. 551-576, 2012.

[6] A. Ivić and J. Wu, "On the general additive divisor problem," Proceedings of the Steklov Institute of Mathematics, vol. 276, no. 1, pp. 140-148, 2012.

[7] G. Coppola, "On the Selberg integral of the $k$-divisor function and the $2 k$-th moment of the Riemann zeta-function," Institut Mathématique. Publications. Nouvelle Série, vol. 88, no. 102, pp. 99-110, 2010.

[8] A. Ivić, "The general additive divisor problem and moments of the zeta-function," in New Trends in Probability and Statistics, vol. 4, pp. 69-89, VSP, Utrecht, The Netherlands, 1997.

[9] G. Coppola, "On the modified Selberg integral," http://arxiv .org/abs/1006.1229.

[10] G. Coppola, "On the Correlations, Selberg integral and symmetry of sieve functions in short intervals, III," http://arxiv.org/abs/ 1003.0302 .

[11] G. Coppola, "On the symmetry of divisor sums functions in almost all short intervals," Integers, vol. 4, article A2, 9 pages, 2004.

[12] G. Coppola, "On the correlations, Selberg integral and symmetry of sieve functions in short intervals. II," International Journal of Pure and Applied Mathematics, vol. 58, no. 3, pp. 281-298, 2010.

[13] G. Coppola and S. Salerno, "On the symmetry of the divisor function in almost all short intervals," Acta Arithmetica, vol. 113, no. 2, pp. 189-201, 2004.

[14] J. Kaczorowski and A. Perelli, "On the distribution of primes in short intervals," Journal of the Mathematical Society of Japan, vol. 45, no. 3, pp. 447-458, 1993.

[15] A. de Roton, "On the mean square of the error term for an extended Selberg class," Acta Arithmetica, vol. 126, no. 1, pp. 2755, 2007.

[16] L. Fejér, "Über trigonometrische polynome," Journal für die Reine und Angewandte Mathematik (Crelle's Journal), vol. 146, pp. 53-82, 1916.

[17] B. Green, "On arithmetic structures in dense sets of integers," Duke Mathematical Journal, vol. 114, no. 2, pp. 215-238, 2002.

[18] H. Iwaniec and E. Kowalski, Analytic Number Theory, vol. 53 of American Mathematical Society Colloquium Publications, American Mathematical Society, Providence, RI, USA, 2004.

[19] S. A. Amitsur, "Some results on arithmetic functions," Journal of the Mathematical Society of Japan, vol. 11, pp. 275-290, 1959.

[20] J. P. Tull, "Average order of arithmetic functions," Illinois Journal of Mathematics, vol. 5, pp. 175-181, 1961.

[21] G. Coppola and M. Laporta, "A modified Gallagher's Lemma," http://arxiv.org/abs/1301.0008. 


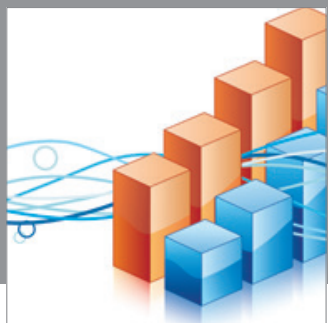

Advances in

Operations Research

mansans

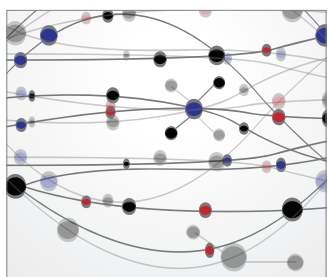

The Scientific World Journal
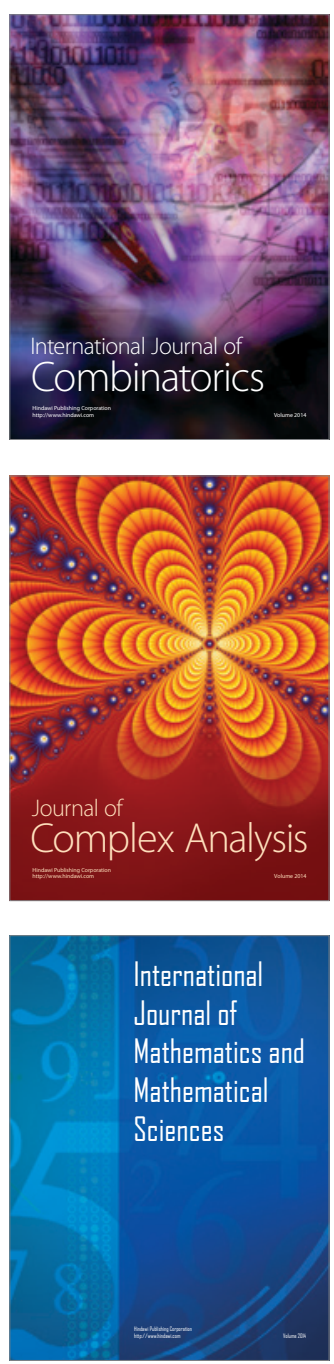
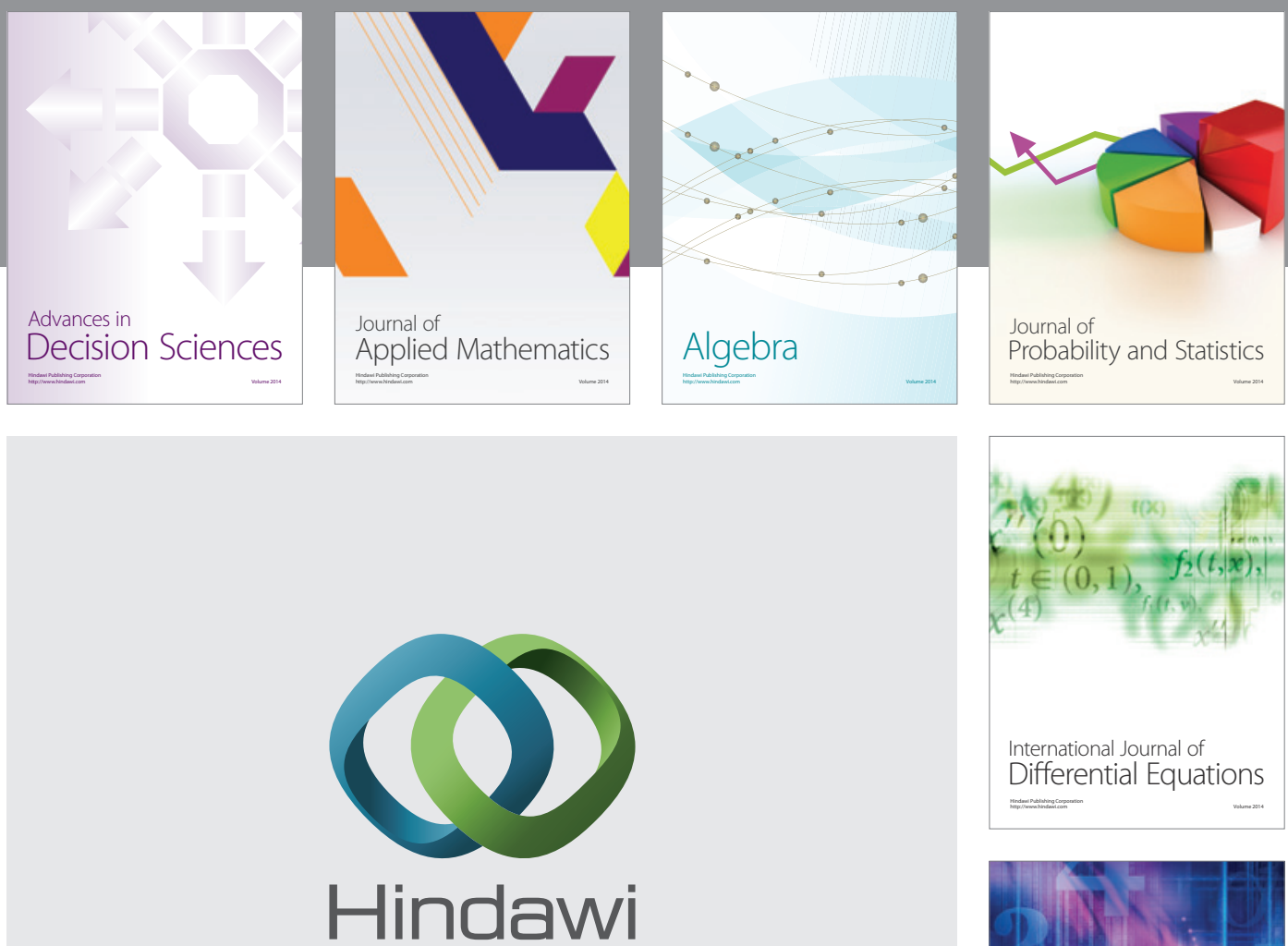

Submit your manuscripts at http://www.hindawi.com
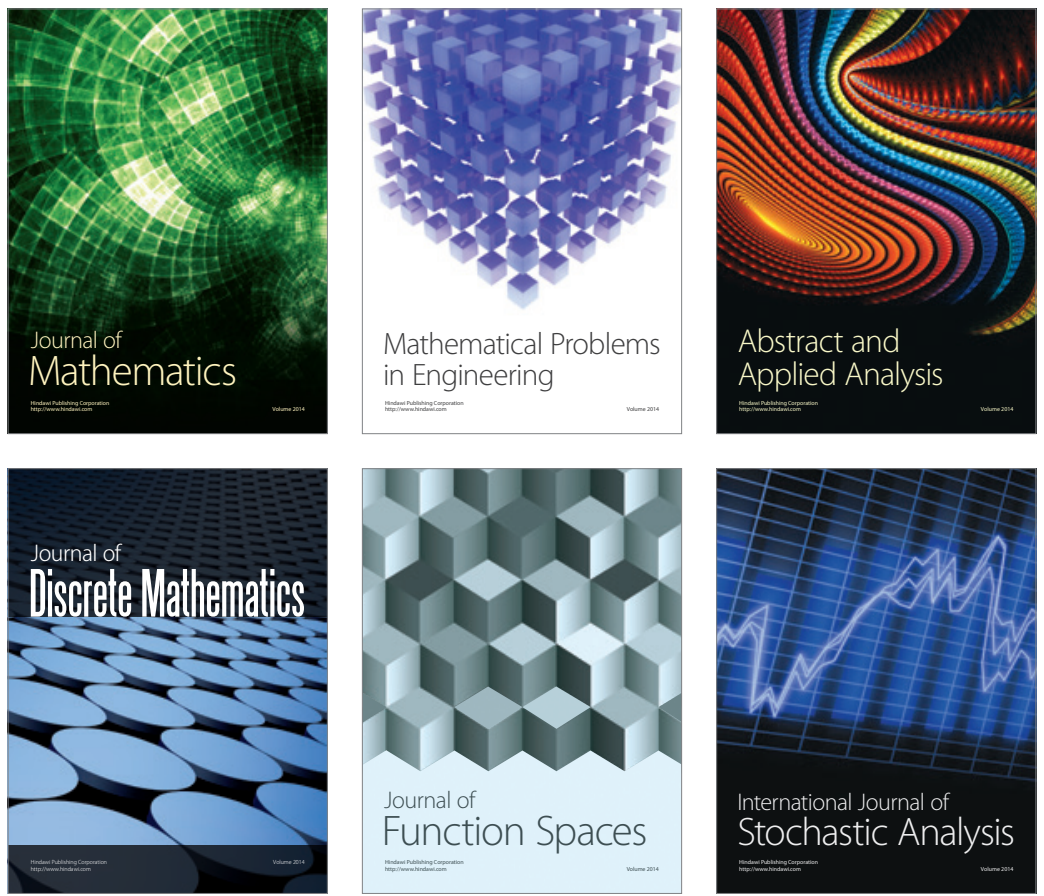

Journal of

Function Spaces

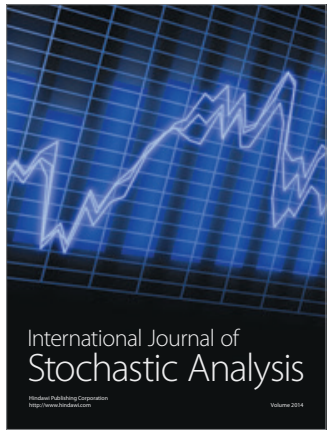

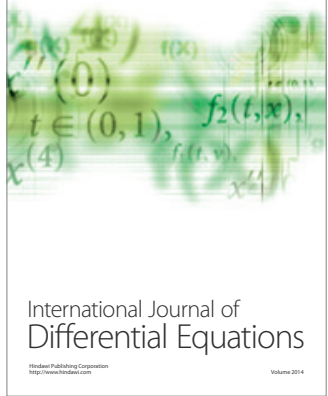
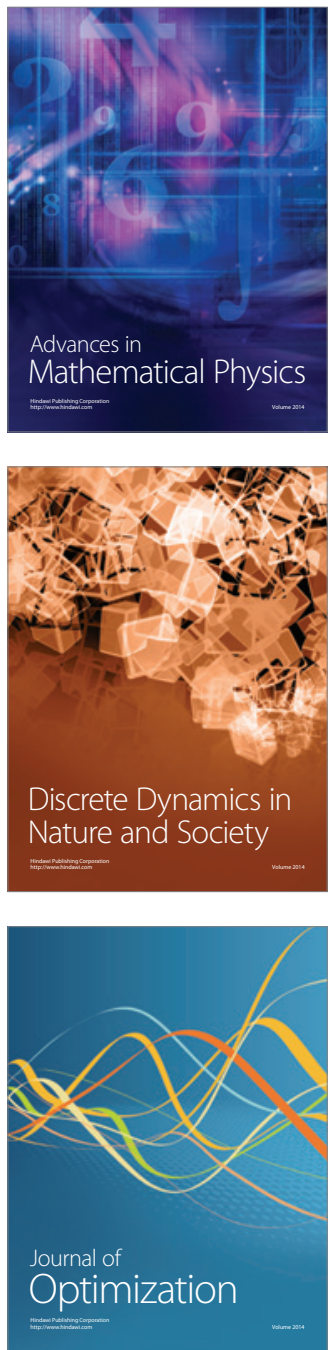